\title{
Cross-linguistic Study on Visual Syntactic Priming of Imbalanced Chinese-English Bilinguals
}

\author{
Rouhua Wang \\ Foreign Language College \\ Northeast Normal University \\ Changchun, China 130024 \\ Changchun Institute of Technology \\ Changchun, China 130022
}

\author{
Yongbing Liu \\ Foreign Language College \\ Northeast Normal University \\ Changchun, China 130024
}

\begin{abstract}
Syntactic priming refers to the phenomenon in which an individual uses a previously used sentence to produce output when producing and understanding a sentence. The initial study of syntactic priming started in the English language and gradually spread to other languages, and went deeper into cross-language syntax. However, the current crosslinguistic syntactic priming rarely involves Sino-Tibetan language families to which Chinese belongs; there are disagreement on syntactic priming in sentence production and sentence comprehension; there are different views on the role of verbs in syntactic priming; and on the level of bilingualism. The role of the role played in syntactic priming is not clear. In this study, two experiments were designed to solve the above problems. The visual prime and auditory prime methods were used, and the image description paradigm was used to initiate bilingual bi-directional syntactic study of the same batch of Chinese English forty-six subjects. The study contains four sentence patterns that are most commonly used in syntactic priming studies: active and passive sentences, double-object sentences, and preposition. The following results are obtained: 1. there is a bidirectional cross-channel syntax priming between English and Chinese; 2.Verb dependence has a sentence-specificity and may expand to a larger cognitive burden. In other sentence patterns, the possibility of such expansion decreases as the frequency of use of the sentence itself increases; 3. As the level of English increases, the efficiency of syntactic priming increases.
\end{abstract}

Keywords-syntactic priming; English level; prime channel; verb repetition

\section{INTRODUCTION}

The mechanism of sentence processing has long been concerned by experts on the field of psychologists and artificial intelligence. Syntax prime is considered to be an excellent method of studying syntactic processing mechanisms. The study of syntactic primitives began with Bock's (1990) study of the production of two types of syntactic in English. Bock examined the passive and passive sentences in English, as well as the Double-Object (DO) and Prepositional-Object. (PO Syntax for short) Syntax prime. Example sentences are as follows:

Active sentence: A drunk is pushing an old man
Passive sentence: An old man is being pushed by the drunk

DO sentence: The boy sends girl the flower

PO sentence: The boy sends the flower to girl

In the experiment, a prime sentence was produced by the main tester, asking the participant to read the sentence after the prime of the reading to present a picture, requesting that the picture was described in a trial language. The experimental results show that the previously presented prime sentence has a significant initiating effect on subsequent speech production. Using PO sentences and DO sentences as an example, using a PO sentence as a priming sentence means that the subject is more likely to describe the picture using the structure of the PO sentence, and this is also true of the DO sentence and other sentences structures. With the deepening of the study, Bock et al. conclude that the syntactic generation has no relevance to the relationship between the surface of the sentence and the prime of syntax. It is also not the result of rhythm or lexical repetition. It is independent of the stem and meaning level, and is the priming and target sentence. The same syntactic structure determines.

Later researchers were interested in the priming of crosslanguage syntax, suggesting that the transition to languages is more helpful in revealing the essence of sentence processing. The first cross-linguistic syntactic priming studies began in Loebell and Bock (2003) in German and English. Subjects were asked to repeat the sentence in German or English and then describe the picture in another language. The experimental results verify the syntactic priming of the lattice structure (PO sentence and DO sentence) between English and German, but the syntactic priming of the passive structure of the two has not been found, and this has also become the concern of later researchers. The priming of passive sentences in syntactic priming was discovered in the study of Spanish in English by Hartsuiker et al. (2004), validating the hypothesis that Spanish and English share the same passive node, and more ecologically effective confederation scripting techniques. It was also widely used later. 


\section{RESEARCH REVIEW}

The researchers summarized the overall status of research on syntactic priming and its research paradigm and its variants. Secondly, the pair of examples is: the degree of life and syntactic priming, sentence comprehension and sentence production, and syntactic priming in other languages. Phenomenal and other researcher's focus and progress are described. Third, the necessity and research status of crosslanguage syntax priming are summed up. Finally, domestic and foreign research is combined to summarize the mechanism behind syntactic priming and inter-lingual syntactic priming.

The study of syntactic priming started with Bock's (1990) study of the production of two types of sentence patterns of English. Bock examines the syntax priming of doubleobjective (DO-sentence) and prepositional-object (POsentence) sentences in English.

In the experiment, the host tries to show the prime sentences of different sentence patterns. After the test sentence is read, the examinee presents a picture and requests that the content of the picture was described in the spoken language. The experimental results show that the different types of initiating sentences presented previously have a significant initiating effect on subsequent speech production. Using PO sentences and DO sentences as an example, using a PO sentence as a priming sentence means that the subject is more likely to describe the picture using the structure of the PO sentence, and this is also true for the DO sentence and other sentences structures. Subsequent research by Bock (1990) also found that even if the preposition is different from the target sentence, the prime effect also exists. For example, the prime sentence "the girl baked a cake for her boyfriend" is against the official lady handed a form of the man. This target sentence has a prime effect. However, the priming effect cannot occur when the experimenter uses a similar rhythm sentence as the priming sentence and the target sentence. The above results were also verified in subsequent experiments (Bock \& Loebell, 1990; Branigan, Pickering, \& Cleland, 2000). A Bock et al. concluded that syntactic priming did not depend on the relationship of the sentence surface. The result of rhythm and lexical repetition, which is independent of stem and word meaning levels, is determined by the same syntactic structure of the initiating and target sentences.

\section{QUESTION PROPOSES}

The researchers question the uncertainty of their own research: (1) whether there is a cross-language syntactic priming between Sino-Tibetan and Indo-European languages; (2) Syntactic understanding and sentence production of syntactic priming Whether there is a difference; (3) Whether the verb dependence phenomenon only exists in the syntactic priming of sentence comprehension; (4) The effect of the second language level on the effect of syntactic priming is positive or negative.

Syntactic priming has been validated in experiments in multiple countries and languages, and researchers' interest has begun to shift to cross-language syntax priming. Cross- language syntax priming refers to the phenomenon of syntactic priming when the prime language and target language are in different languages.

The first cross-linguistic syntactic study started with Loebell and Bock (2003) using German and English Language research. Similar to the original syntax-initiated picture description paradigm, subjects were asked to repeat sentences in either German or English and then describe the pictures in another language. The experimental results verify the syntactic priming of the lattice structure (PO sentence and DO sentence) between English and German, but the syntactic priming of the passive structure of the two has not been found, and this has also become the concern of later researchers. The priming of passive sentences in syntactic priming was discovered in the study of Spanish in English by Hartsuiker et al. (2004). This experiment validates the hypothesis that Spanish and English share the same passive node, and the more ecologically valid ally Scripting technology has also been widely used later. Similar results have also been found by other scholars (Meijer \& Fox Tree, 2003). Schoonbaert et al. (2006) found that by the two-way primeup of Dutch and English, there is a syntactical prime phenomenon from Dutch to English. The equivalence between translation verbs can effectively promote the priming phenomenon, but from English to Dutch In the prime experiment of the language, no prime phenomenon of equivalence translation verbs was found. In the same year, Desmet et al. (2006) also investigated the syntactic priming between Dutch and English. Among them, the study subjects were all bilingual and highly skilled users (including Dutch as mother tongue and English as second language). The research paradigm was sentence complementation. However, in the research paradigm, researchers used ambiguous attributive clauses to conduct research. It was found that the complement of highly attached Dutch sentences played a significant role in the completion of the English sentence in the high attachment form of the later period of the test.

Whereas previous studies have been conducted on the languages of the Indo-European language system (the most widely spoken languages in the world), the similarity between syntactic structures of the same language system cannot be ignored as a variable to explain the syntactic priming. Some researchers have studied this. Arai et al. (2007) selected foreign students whose native language is Japanese to study in Edinburgh to use the sentence recall method to conduct research. It was found that the repetition of verbs can effectively stimulate syntactic priming in sentence comprehension, and that the more spontaneously enriched sentences have a greater amount of priming. . Shin et al. (2009) verified the existence of cross-language syntactic priming through the activation of Korean and English grammatical structures, and concluded that the sequence of arguments had no effect on syntactic priming results.

Hartsuiker et al. (2004) developed a cross-language version of a syntactical lexical representation model based on the syntactic characterization model of the word proposed by Dickering et al. (1998) after using syndication scripting techniques to study syntactic priming between Spanish and 
English. First, Hartsuiker introduced language nodes on the basis of the original lemma node, category node, feature node, and combinatorial node of the entry layer. 2002; Van Heuven e. al (1998), used to activate different language classifications. Take picture 4 as an example, hit and in English. The corresponding verbs for base of Spanish are gulper and poseur. They are both connected to Verb (category node) as verbs and to Active and Passive nodes (joint information nodes) at the same time. In addition, they belong to the conceptual layer (vocabulary). It exists on the form of a concept here, without involving other concrete forms of the same node HIT (X, Y ) and CHASE (X, Y ). So when " hit" in "Someone hit the man" is activated, the "gulper" of Spanish is activated at the same time through the concept layer HIT CX , Y ), while the category node "Verb" to which "hit" belongs is activated. The joint information node "Active" is activated, so that if there is a "gulper" at the prime of the next target sentence, the subject is likely to answer with the same sentence, and the model also explains if the verb of the target sentence is inconsistent. When the prime phenomenon occurs, that is, the joint information node to which " hit " belongs is used by the verb of the target sentence, so even if there is no verb repetition, syntactic priming will still occur, and verb repetition will enhance the effect of syntactic priming conceptual terms Activation .

\section{FORMAL RESEARCH}

This part contains two experiments. In Experiment 1, the researchers used the self-produced materials to examine the Chinese-English and Chinese cross-language syntactical primes in English and Chinese in the two pairs of opposing sentences: active and passive, double-objective and prepositional. The level of verbs and the manner in which sentences were produced was investigated at the same time. The researchers simultaneously conducted in-depth discussion of each sentence pattern of each direction. In experiment, the same subjects as in experiment were selected to use new materials under auditory presentation. The variables and experimental methods are consistent with experiment one.

The experimental study on the prime of Chinese-English Bilinguals about Cross-linguistic Bi-directional Syntax. The first experiment mainly explores the two-way four-sentence bidirectional syntax stimuli under visual priming dynamic effect.

\section{A. Experiment - Research Purpose}

Explore whether there is a phenomenon of crosslanguage syntactic priming under visual priming and explore the role of variables such as verb level, output style, and English level in bidirectional priming. The purpose of this research study is as follows:

- Under the unified background, to explore whether there is a bilingual bi-directional syntactic priming phenomenon;

- Whether sentence production and sentence comprehension have differences in the prime of
English and Chinese double-sentences methods, exploration verbs, bilingualism and other factors play a role.

\section{B. Experimental Method}

1) Experimental design: Based on the points raised in the question, Experiment examines the prime phenomenon of Chinese-English and Chinese-English bi-directional syntax under visual priming. In each direction, the study uses the English level (2) X syntax forms (4) X verb level

(2) $X$ generation mode (2) mixed design, in which the English levels is divided into low and high level two groups of subjects, syntactic forms (active and passive, double-bin and prepositional), verb levels (consistent, inconsistent)), how to produce (understand, produce) for the test variables. In the experimental design, the variables are manipulated as follows: (1) After the syntactic format is prepared by the main test, it is sent to the English graduates of the 8th level and the Chinese graduates to jointly determine, for example, the test preparation "he took the book." or "He took it away. The book "consistent with the SVO structure sentence (subject + transitive verb ten noun) is regarded as an active sentence; similarly if there is a "by" (English) or a Chinese "accepted" word is considered as a passive sentence; "He give me a book" or "He gave me a book" is in line with the verb ten. Someone + something is regarded as a doubleobject statement, and if a verb appears as a certain thing + a preposition (giving) to someone, it is regarded as Bin sentences; (2) The verb levels are determined jointly by graduate students of English majors and graduate students of the Chinese language. For example, "give" and "give" are considered as consistent verbs and words such as "teaching" and "returning" are considered. It is an inconsistent verb; (3) understanding and production are arranged by the main test in advance, all the items required for selection are for understanding, and all requirements for the creation of sentence items according to the given verb.

2) Experiment test: From the University of Jilin Province, 40 students were selected, of whom twenty were freshmen or sophomores, with an average age of 18.98 years, all passing the English Test Band 4, and the other 20 graduate of an average age of 27.05 years. All passed the English Level 6 test. All subjects were normal vision or normal after correction.

3) Experiment materials: 108 black-and-white images were selected from the web by using simple text or cartoon pictures, of which 36 were used as prime images, 36 as target 1 images, and 36 as target 2 images. There is one trail for each subject's intrinsic variable (each trail corresponds to 3 pictures), a total of 2 (answer method) X4 (syntax) X2 (verb level) totals 16 trails, and a total of 32 trails in both language directions There are also 4 trails as exercises. There is a primeup picture for each trail. The picture below corresponds to one language sentence, and the second picture serves as the target picture. There may be two cases: The first picture shows three options described in another language. Among them, two options are the correct 
description of the picture (but different sentence syntax, such as: active or passive, double-object or mediation), another option is the wrong description of the picture; in the second case there will be a Mark a picture with another language verb. The third picture is based on the second picture. If the second picture is an option picture, the third picture is marked with a verb picture. If the second picture is a verb picture, the third picture is an option picture. All third pictures use the same language as the primeup picture. The English sentences of all materials in the experiment were prepared by graduate students of the English majors (and invited the students of Level 4 for oral translation and selected by the Specialized Graduates for approval). The Chinese sentences were reviewed by the graduate students of the Chinese University, and the experiments were conducted in the E-prime environment. Run and record. When selecting pictures, the researchers considered that the characteristics of the experiment were based on the prior selection of verbs to select pictures. The picture content did not make special provisions. In principle, as much as possible to ensure that the scene between each group of trail images is inconsistent, even if the verb is consistent in the horizontal trail of three The scenes described in the pictures are also different. There are certain similarities in the experimental trail scenes, but the verbs used are also guaranteed to be inconsistent.

\section{RESULTS AND ANALYSIS OF EXPERIMENT}

There were forty people in the experiment. Two of the four students in the actual operation experienced operational errors that made the data unusable, so the actual results included 18 four-level subjects and 20 sixth-level subjects.

Chinese-English Active Sentences and Passive Sentences under Visual Activation Researchers first compared the activation effects of the active-sentence and passivesentences in the Chinese-English direction. The results are shown below.

\section{A. Chinese-English Active Sentence Prime}

SPSS21.0 was used for data processing. The English level was taken as the subject's independent variable, verb level and output pattern as participants' internal variables. The priming score was used as the dependent variable to carry out the mixed design analysis of variance, and the scoring direction factor was added (forward calculation. Subinverse scoring is used as an independent variable, that is, if the prime is successful, it is a positive score. If it is not started successfully, it is scored on the reverse score level. The purpose of comparing positive and negative scores is to validate syntax priming from absolute values. The reverse score is essentially a dummy variable. In this case, the main effects of considering the verb level, output mode, and English level separately are meaningless. Each factor must interact with the score direction to prime.

The experimental data analysis results show that the prime effect is significant, $\mathrm{F}(1,37)=189.28$,, $\mathrm{p}=$. 000 , the positive score is greater than the reverse score, the active sentence is more inclined to produce the active sentence. . Among them, the verb level, output mode, and scoring direction have significant interactions. $\mathrm{F}(1,37)=23.54, \mathrm{p}=$. 000. After further analysis of the simple effects of the three, it was found that except when the inconsistent verbs were used to understand the assignments, $\mathrm{F}(1,37)=7.94, \mathrm{P}=.338$, the scoring direction shows positive scores greater than reverse scores at each level of verbs and output methods.

Ignoring the scoring direction, simply considering the composition of the sentence components that have been started, the following results are obtained: The main effects of verb level are significant, $\mathrm{F}(1,37)=12.27, \mathrm{P}=.001$, verbs are consistent The resulting active sentences are greater than the active ones produced by inconsistent levels of verbs; the main effects of the output methods are significant, $\mathrm{F}(1,37)=$ 7.61, $\mathrm{p}=$. 009, more active sentences than under comprehension Active sentence under conditions. The interaction between verb level and output style is significant, $\mathrm{F}(1,37)=13.00, \mathrm{p}=.001$. In the understanding of homework, the consistent verb produces more active sentences than the inconsistent verbs; under the inconsistent verbs, it expresses The efficiency of the job is greater than understanding the job.

\section{B. English and Chinese Passive Sentences Prime}

Considering the direction of scoring factors, experimental data analysis found that there is no statistical difference in the number of passive sentences generated between passive and non-passive sentences. $\mathrm{F}(1,37)=3.239$,, $\mathrm{p}=.089$ The interaction between the production method and scoring direction was significantly $\mathrm{F}(1,37)=9.729, \mathrm{p}=0.005$. Further simple effect analysis found that in the expression job, the positive score was greater than the reverse score, $\mathrm{F}(1$, $37)=8.25, p=.011$, that is, the effect of the syntax prime is significant when the participant is required to perform the expression operation under passive prime.

Neglecting the direction of scoring factors, the following results were obtained by analyzing the data in Table 2 . The main effects and interactions of all factors were not significant, and passive sentences were evenly distributed under various factors.

\section{Summary and the Significance of This Study}

Exploring the cross-linguistic syntactic priming between Indo-European and Sino-Tibetan languages is an appropriate enrichment and complement to cross-linguistic studies; at the same time, experimenting with traditional research sentences in a relatively uniform context relies on verbs appearing in previous studies. Different comprehension mechanisms of the output, second language level and other cross-language syntactic priming factors can be compared to get convincing conclusions; cross-language syntactic priming studies can improve the teaching methods of English in our country.

The prime effect of the visual English-passive and passive sentence is significant; the active verbs and expression work in the active sentence all have advantages; the inconsistent verbs appear in the passive sentences and the 
phenomenon of understanding the dominant operation; the English level has no effect on the priming of the active and passive sentences.

\section{CONCLUSION}

This research is in light of the results of formal experiments. It addresses the cross-language syntactic priming (existence), sentence comprehension and sentence production differences (absence), verb dependence (sentence specificity), and English proficiency levels. The impact of cross-language syntax priming (positive) was explored in depth; the specificity of sentence patterns was introduced into the scope of the study, and the mechanism of syntactic priming (one dominant language) was discussed.

The above experimental results suggest that syntactic specificity as a variable that cannot be ignored in syntactic priming experiments should be taken into account in future experiments. At the same time, no matter what the sentence pattern, Chinese-English and English-language bi-lingual primes all show obvious syntactic priming effects, which proves that the syntactic priming has existed in each sentence pattern between Sino-Tibetan and Indo-European languages.

The English level has less significant effect on the syntactic priming effect, but with the deepening of the experiment, the researchers found that the influence of English proficiency on syntactic priming: 1. The impact on English and Chinese is more than English and Chinese, and the English level is The interactions between research variables mostly appear in the direction of Chinese-English; 2. The impact on auditory priming is more than the impact on visual priming. The English level in auditory priming participates in the interaction with other variables earlier; 3 . The influence of uncommon sentences is more than that of common sentences. The most frequently affected by English is passive sentences and double-object sentences.

According to the above rules, the researchers believe that the influence of the English level on syntactic priming is more pronounced at the time of sentence generation, so that Chinese-English is greater than Chinese-English results. This also suggests that the understanding is more application of Chinese. Thinking rather than English thinking, which is also more consistent with the dominance of the phrase, recent studies have also shown that local language experience has an effect on syntactic priming (Nitschke etx1., 2014); secondly, auditory activation itself does not magnify subjects. The absolute difference in the level of English (no difference in visual and auditory activation), but it can be used as a magnifying glass to examine the level of English proficiency of the subjects, so that the difference between the two can be better displayed, at least for Chinese unbalanced English learners. Points should be taken into account; third, the gap in English proficiency is more likely to occur in less frequently used sentences, but infrequently used sentences have a low prime rate, and the absence of a comparable number may cause failures in the experiments to prime.

Researchers believe that based on the results of the research, they have verified several theoretical findings that the above-mentioned multiple theories actually show us a syntactically initiated imbalanced development process. There are relatively few subjects who are balanced in Chinese bilingualism and it is still unclear whether the results of their research can be extended to the majority of English learners. However, if students are selected from a wide range of unbalanced English learners, and then the commonly used and infrequent sentences in second language learning are taken as the scale of different stages of different syntactic development, we can actually see the syntactic development in one subject. A rough picture of the entire process. This study actually provides such a channel to verify the theory.

This part is the conclusion part. The researchers got the following conclusions:

- There is a bidirectional cross-channel syntax priming between English and Chinese;

- Verb dependence has a sentence-specificity, and it may expand to other sentence patterns as the cognitive burden increases. This possibility of expansion decreases with the increase of the frequency of the use of sentence patterns.

- As the level of English increases, the efficiency of syntactic priming increases;

\section{REFERENCES}

[1] Arai M,Gompel RP Scheepers C. Priming Ditransitive Structures in Comprehension[J]. Cognitive Psychology, 2007, 54(3): 218-250.

[2] Bock K,Loebell H. Framing Sentences.[J]. Cognition, 1990, 35(1): 139

[3] Branigan, H. P., Pickering, M. J.,\&Cleland, A. A. (2000). Syntactic co-ordination in dialogue. Cognition, 75, B13-25.

[4] Desmet T., Declercq M. Cross-linguistic Priming of Syntactic Hierarchical Configuration Information[J]. Journal of Memory and Language, 2006, 54(4):610-632.

[5] Hartsuiker, R. J., Pickering, M. J.,\&Veltkamp, E. (2004).3 between languages?Cross-linguistic syntactic priming in Spanish-English bilinguals. Psychological Science,15(6), 409-414.

[6] Loebell, H.,\& Bock, K. (2003). Structural priming across languages. Linguistics, 41 (5; ISSU 387),791-824

[7] Meijer PJA,Tree JEF. Building Syntactic Structures in Speaking: a Bilingual Exploration[J].Experimental Psychology (formerly “zeitschrift Fur Experimentelle Psychologie"),2003, 50(3): 184-195.

[8] Nitschke S,Serratrice L,Kidd E. The Effect of Linguistic Nativeness on Structural Priming in Comprehension[月.Language, Cognition and Neuroscience, 2014, 29(5): 525-542.

[9] Salamoura A.,Williams J. N. (2006). Lexical activation of crosslanguage syntacticpriming. Bilingualism: Language and Cognition, 9, 299-307.

[10] Van Heuven, W.J.B., Dijkstra, T.,\&Grainger, J.(1998). Orthographic neighborhood effects in bilingual word recognition.Journal of Memory and Language, 39,458-483. 